\title{
Arbor
}

\section{Viejos recuerdos en torno a la construcción del Instituto Cajal y de Microbiología en 1950}

Miguel Fisac

Arbor CLX, 631-632 (Julio-Agosto 1998), 333-341 pp.

En este artículo se presentan algunos de los recuerdos del autor sobre los principales personajes en la fundación del Consejo Superior de Investigaciones Científicas, asi como su participación como arquitecto en algunos de los edificios más emblemáticos del mismo.

Se me piden unas notas, para el número especial que la Revista Arbor dedica al Instituto Cajal.

Comenzando por el principio, diré que a Don José M. ${ }^{\text {a }}$ Albareda lo conocí en Barcelona en octubre de 1937, cuando llegué con un amigo que fue a recogerme, después de permanecer un año escondido, entre el tejado y el techo de mi casa, en mi pueblo, en La Mancha, para liberarme de la persecución, a muerte, de que era objeto mi familia.

Un mes después de mi llegada a Barcelona, un pequeño grupo de siete amigos, algunos, no todos, del Opus Dei, con Mons. Escrivá, marchamos hacia Andorra en autobús y estuvimos otro mes esperando a los guías en el bosque de Rialp. Y después de cinco extenuantes noches, llegamos a Andorra, y por Francia, a Fuenterrabía.

Mi inmediata incorporación de soldado, al frente de Teruel, como chofer, ya que era obligado al tener yo carnet de conducir, hizo posible que yo pasara repetidas veces por Burgos, en donde José M. ${ }^{a}$ Albareda, con el Sr. Escrivá, vivían en un modesto hotel. Mi amistad con Albareda, 
durante toda esta odisea, se consolidó muy fuertemente. Al regresar a Madrid, Albareda comenzó a estructurar lo que sería el Consejo Superior de Investigaciones Científicas.

Nuestras mutuas convicciones cristianas de la vida, hicieron que compartiera con él su deseo de que de forma arquitectónica, quedara plasmada, en el nuevo núcleo de edificios de Investigación que se pensaba construir, una iglesia con advocación al Espíritu Santo.

Los edificios dependientes de la Junta para Ampliación de Estudios, pasaron a la nueva ordenación del C.S.I.C. y entre ellos, había un simplicísimo edificio de planta rectangular, de paredes y techo enyesados y pintados al temple liso, como salón de actos, y Don José M. ${ }^{a}$ Albareda me indicó que, tal vez, podría ser adaptado a Iglesia del Espíritu Santo.

Visité el edificio y me pareció que no había nada que pudiera tener valor arquitectónico, ya que se notaba una cierta provisionalidad y, sin embargo, el claustro adherido a él, sí que, dentro de su sencillez, me pareció precioso, realizado con toda sabiduría, por el gran arquitecto Arniches.

Le comenté mi opinión a Albareda y me dijo que podría, con mi propuesta, aprovechando las paredes de cuatro metros de altura, hacer un anteproyecto de adaptación para lo que podría ser la Capilla del Espíritu Santo, sin tocar para nada el claustro, como así se hizo.

Indiqué en la memoria, que creo le gustó al Ministro Sr. Ibañez Martín, que con un presupuesto de un millón trescientas mil pesetas, incluida la decoración, el altar y el mobiliario, etc., podría realizarse la obra cuyo anteproyecto se aceptó.

Este proyecto, que gustó, se me adjudicó, como digo, por amigable intervención de don José M. ${ }^{a}$ Albareda miembro del Opus Dei, como yo en aquel tiempo.

Los encargos posteriores que recibí después del Ministerio de Educación, fueron consecuencia de que el $\mathrm{Sr}$. Ministro estuviera muy satisfecho de la marcha y calidad de las obras de la Capilla del Espíritu Santo que é1 visitaba casi todas las semanas.

Cuando yo salí de la Escuela Superior de Arquitectura y también mis otros nueve compañeros de curso, todos tuvimos trabajo ese mismo mes: ya que había mucho trabajo y pocos arquitectos.

A mí, el Director General de Arquitectura, Don Pedro Muguruza, me había invitado el año final de mis estudios a que fuera a su Estudio del Ministerio a trabajar con otros dos compañeros: Cabrero y Aburto, principalmente en los proyectos de reconstrucción de la ciudad de Santander y de un barrio de pescadores. $\mathrm{Y}$ al terminar la carrera, me 
Viejos recuerdos en torno a la construcción...

dijo que podía quedarme donde quisiera de las diferentes entidades oficiales que el controlaba de arquitectura y urbanismo, pero yo preferí desarrollar mi profesión por libre. Doy todos estos detalles, porque sé que, malévolamente, se ha dicho que mi posterior trabajo profesional se debía a la ayuda que había recibido del Opus Dei. Cosa totalmente falsa, entre otras razones, porque el Opus Dei, era en aquellos tiempos, una entidad totalmente desconocida, sin aprobación cánonica diocesana y sin influencia social y política.

Antes de terminar la Iglesia del Espíritu Santo, recibí del Sr. Ibáñez Martín el encargo de proyectar el Edificio Central del Consejo de Investigaciones Científicas y los edificios de entrada a la Plaza: los Institutos de Geología y Geografía (Geos), la Urbanización de esa plaza y el Instituto de Óptica.

Después, en una parcela triangular y residual resultante de la continuación de la calle de Velázquez esquina a Joaquín Costa, se pensó en construir un edificio que albergaría el Instituto Cajal y el de Microbiología. Dos Institutos que necesitaban un edificio adjunto para cría y seguimiento en trabajos de investigación con ratones, ratas, cobayas, gatos, perros, etc.

Don José M. ${ }^{\mathrm{a}}$ Albareda me explicó que era esencial en esos Institutos la óptima instalación de estos animales: su cría, su situación incontaminada al comienzo de una experiencia, su seguimiento a lo largo de este trabajo, su absoluto aislamiento, exento tanto de su contagio en uno u otro sentido, así como su eliminación final con todas las garantías profilácticas.

Se me concedieron 8.000 pts. como bolsa de viaje, suficientes para realizar, con austeridad, el recorrido que me habían marcado, y con muchas cartas dirigidas a los Directores de los Centros que tenía que visitar.

\section{Viaje por Europa}

El día 19 de Octubre de 1949 comencé el viaje para visitar los diferentes centros, en los que quería estudiar las instalaciones de animales de experimentación.

En Suiza, aunque llegué directamente en avión desde Madrid a Ginebra, pasando después en tren, por Berna y Zurich, el objeto de estudio era la visita a las instalaciones farmacéuticas de Basilea: Hoffam la Roche, Ciba y Sandof. Desde allí, en avión a París, para visitar el Pasteur y desde allí, también en avión, a Estocolmo para estudiar las instalaciones del Instituto Novel de Biología, y casi en el Círculo 
Polar Ártico, más arriba de Uppsala, en Ultuna, donde se acababa de inaugurar el nuevo Instituto de Microbiología.

Después, en tren, pasando por Gotteborg y Malmö, de gran interés arquitectónico para mí, fui a Copenhague, Amsterdam y Bruselas, regresando a Madrid, el 15 de Noviembre.

Para mí, profesionalmente, fue un viaje interesantísimo y terminé siendo un experto en la estabulación de animales de experimentación.

Con todos esos datos y los programas que me proporcionaron el Dr. Rodríguez Candelas para el Instituto Cajal, del que era director y el Prof. D. Lorenzo Vilas director del de Microbiología, pude desarrollar los primeros Anteproyectos que cumplieran las condiciones requeridas para este tipo de edificios.

\section{Criterios de composición del edificio}

La forma triangular del solar, con vértice entre las calles Velázquez y Joaquín Costa, y teniendo que edificar un edificio que había de cumplir, como programa, dos bloques independientes, y hasta con entradas y enlaces verticales: ascensores y escaleras, también independientes, pero que tuvieran en cada planta enlace a un tercer bloque de servicio de todas las instalaciones requeridas para cría y estabulación de animales de experimentación, y definían tanto la forma y abcesos y eran tan simétricos los condicionantes que el resultado no podía ser más a la medida de su obligada composición. El edificio de animales de experimentación se instalaba en la esquina, con una ordenación de huecos y estructura mucho más cerrada y dos bloques adheridos laterales, a una y otra calle, el Instituto Cajal a la calle de Velázquez y el de Microbiología a la de Joaquín Costa. Para la circulación interior era conveniente dejar diáfanas estas dos entradas con el jardín interior y también de más grato efecto urbano que el de un gran edificio cerrado y, como el uso de estos edificios era para laboratorios, el módulo por puesto de trabajo interior en ellos, obligaba a un tipo de estructura de acuerdo con ese requerimiento y también a una separación y apertura de unas ventanas determinadas.

\section{Consideraciones constructivas y estéticas}

Al comenzar el proyecto de los Institutos Cajal y de Microbiología yo contaba con ocho años de experiencia profesional muy activa. Había 
Viejos recuerdos en torno a la construcción...

realizado la Iglesia del Espíritu Santo, y todo un conjunto de edificios de Dirección del C.S.I.C., ordenación de su plaza, intervenciones fuera del C.S.I.C., viviendas unifamiliares y plurifamiliares de pisos, de restauración de viviendas, construcción de dos Residencias de estudiantes en Barcelona y Santiago de Compostela, etc.

$\mathrm{La}$ inauguración del conjunto de la "colina de los chopos" para el C.S.I.C., casi la hice con mis propias manos, porque las obras se realizaron por administración directa (entonces no se podían realizar subastas). Tuve yo que estudiar los proyectos y la coordinación de escultores, pintores, y realizadores de mosaicos interiores y exteriores, de la fuente de la Plaza, asi como los dibujos de todo el mobiliario, lámparas, alfombras, picaportes, etc.

Todo este conjunto pareció muy moderno y tuvo muy buena acogida de críticos y público.

Como yo siempre he tenido un fuerte sentido, también autocrítico, y a mí no me gustó, o más propiamente, a mí me pareció que aquello no era un camino a seguir aunque, para hacerlo tuve que profundizar desde el principio en el aprendizaje de la arquitectura clásica que me enseñó proporciones, armonía y equilibrio, que me han sido, después, muy útiles.

Pensé entonces que lo que corrientemente se llamaba arquitectura funcional o racionalista que hoy se engloba como Movimiento Moderno y que entonces en España se había abandonado, era cosa de verlo y analizarlo «in situ» $\mathrm{y}$, con los conocimientos prácticos que tenía: estudiarlos.

Comprobé que una cosa era la teoría, y otra muy distinta la práctica, que tenía ante mí unos cuantos latiguillos presentados magistralmente por Le Corbusier, un verdadero genio de la Publicidad y otra muy distinta era la realidad constructiva de aquellos edificios deshumanizados, con muchas deficiencias constructivas y un desprecio al entorno inadmisible.

Claro que al lado de todo eso, Mies van der Rohe, (para mí, el arquitecto más importante del siglo $\mathrm{XX}$ ) ofrecía una arquitectura muy esencial, con una técnica completamente inalcanzable en la situación de entonces de España, y completamente deshumanizada.

La contemplación, entonces, de la arquitectura del norte de Europa, en aquel viaje, visitando instalaciones de animales de experimentación, me proporcionó muchos motivos de enseñanza arquitectónica $\mathrm{y}$, sobre todo, en la minuciosa visita a la ampliación del Ayuntamiento de Gotteborg, de Asplund, (que había muerto hacía nueve años) vi, muy claro, que se podía hacer una arquitectura actual y con una estética 
consecuente con la técnica actual. Una estética sin formalismos ni plástica de receta previa.

Estas enseñanzas las comencé poniéndolas en practica en el Instituto Laboral de Daimiel y luego en el Instituto Cajal. Con un mismo criterio y resultados plásticos completamente distintos, como correspondían a un único planteamiento arquitectónico, pero no formal.

Mi itinerario en el edificio del Cajal: fue repasar con todo cuidado los programas que me habían dado y obtener un organigrama de enlaces y espacios que cumplieran todas las exigencias pedidas. A continuación, estudiar qué estructura demandaban esas obligaciones previstas, llegando a la conclusión, de que en las zonas sin huecos o con muy pocos, lo más económico serían unos muros de ladrillo macizo a cara vista, y así lo hice en el cuerpo de edificio de esquina y en los finales de los dos cuerpos laterales del conjunto.

La salida de emergencia, entonces no se exigía en la Ordenanza de Madrid. Yo terminé poniéndola en el pasillo, en un hueco lateral para iluminarlo, de forma que no deslumbrara, como yo había comprobado que sucedía en iluminación con hueco frontal, pudiendo, a la vez, ser de emergencia en una situación de peligro.

En el resto de los dos cuerpos de edificio laterales, lo más razonable y económico era hacer, una solución de estructura adintelada de hormigón armado. Pero la solución ordinaria, en muchos edificios de oficinas y de viviendas en Madrid, era hacer un cerramiento de ladrillo macizo visto muy pesado. A mí me repugnaba poner un material tan pesado, como el ladrillo macizo, como simple cerramiento y entonces pensé que si en un ladrillo normal hueco doble, una de sus caras se dispusiera con una cierta inclinación se obtendría un goterón que solucionaría la impermeabilización de aquella cara.

Hago un inciso, para comentar que ésa fue mi primera patente que no se ha generalizado más, por mi nula condición empresarial, pero que tanto yo, como algúnos otros compañeros, la ejecutaron con pleno éxito.

Cuarenta y cinco años después y por causa de estar una fachada en una calle rigurosamente orientada a poniente, en las últimos hiladas junto a la cornisa, en la fachada que recibe el color de los rayos infrarrojos de la puesta de sol, algunas piezas se desprendieron en su cara exterior, con peligrosidad para los transeúntes. Esta cuestión que no es nueva, muchos otros arquitectos, y yo mismo, lo hemos sufrido también con otros materiales: mármol o plaquetas, y se puede resolver volviendo a reforzar sus juntas y su superficie con productos incoloros actuales muy prácticos y que con un andamio colgante, hubiera 
Viejos recuerdos en torno a la construcción...

sido de muy poco gasto. El arquitecto al que le han encargado esa reparación y otras reformas, no me ha pedido parecer, sino que todas ellas las ha realizado de la forma más cara y ostensible.

La problemática estética del edificio, como ya comencé a realizarla desde entonces, responde al final de esa especie de marcha analítica, que desde entonces, empecé a realizar en mis proyectos y que continúo haciendo. Y, que consiste, en seguir el itinerario que responde a las preguntas: ¿Para qué se hace este proyecto? ¿En dónde se hace? ¿Cómo se construye? Después queda ese "no se qué" que cada uno, con su peculiar sensibilidad, procura que responda a lo que le parece estéticamente mejor.

En este caso, el problema era, al tratarse de un edificio rigurosamente simétrico y no tener volúmenes distintos, que se creara una tensión en el conjunto, ya que debido a esa simetría resultaba una solución como estática. Aprovechándome de que los huecos de la escalera y de las diferentes habitaciones de estabulación de animales estaban a diferente altura, y suprimiendo uno de los huecos de la planta baja y otro de la parte opuesta del último piso proyecté una ordenación de huecos inclinada lo que motivaba una tensión oblicua. Con un balcón en la terraza, en la parte alta, y una fuente en la baja, en oblicuidad opuesta, se podía obtener un equilibrio dinámico.

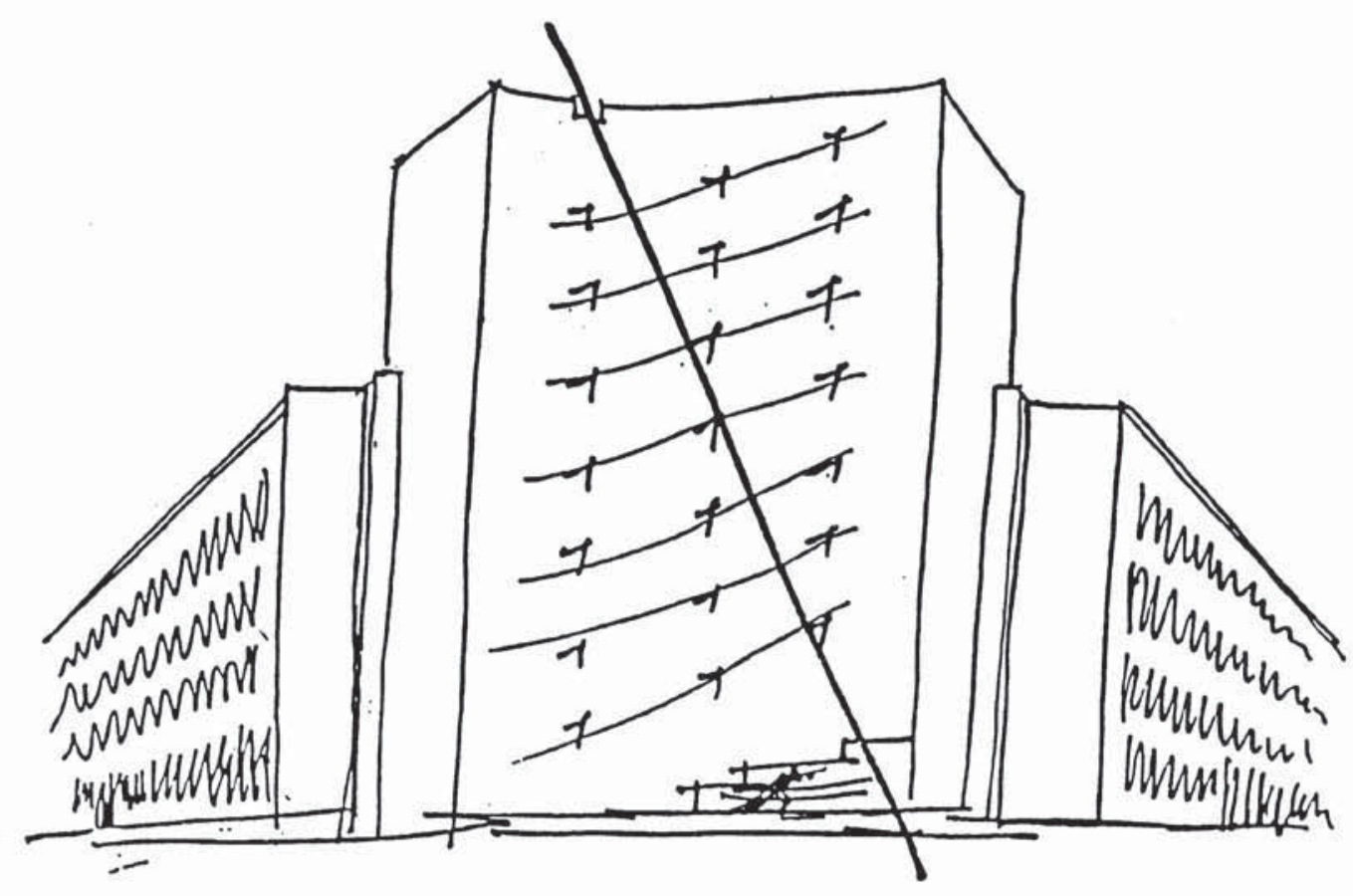




\section{Instalaciones}

En la época en que se proyectó y ejecutó el Edificio Cajal y de Microbiología, las instalaciones, que aparte de las conducciones estéticas necesarias para iluminación, teléfono y las necesarias para su conexión con la red eléctrica, eran escasas y muy primitivas.

Hay que tener en cuenta, que el primer edificio en el que realicé una instalación de aire acondicionado, fue bastantes años después, y ni las personas estaban acostumbradas, ni los aparatos tenían un grado de desarrollo mínimo. Pues el mismo día que recibía una llamada de protesta de algunos empleados que trabajaban en el edificio, porque tenían demasiado calor, llamaban otros protestando que tenían frío.

Aparte de los problemas que había del personal de mantenimiento, me comunicaban que el compresor de frío, tenía un escape de amoniaco, por ejemplo.

En el caso del edificio del que trato, la única instalación que pude montar, además de las eléctricas, fue simplemente un compresor de aire, para que toda la torre de los animales de experimentación y las galerías de los edificios del Cajal y Microbiología consiguieran al abrir las puertas de los diferentes laboratorios, que no pudiera salir el aire que pudiera estar contaminado.

Aunque diseñé una ventana especial, de doble acristalamiento de una pieza y una persiana entre las dos lunas pude comprobar, años más tarde, desde el exterior, que las habían sustituido por otras metálicas de tres espacios y persianas vulgares y habían sacado al exterior unos acondicionadores de aire acondicionado, como en cualquier casa de vecinos de barrio.

Los edificios, como los hijos, cuando se emancipan, pueden decidir, toda clase de modos de entender la estética y también las formas de trabajar.

\section{Otros recuerdos y el Museo Cajal}

Un muy grato recuerdo mantengo de la época en la que fue director del Cajal Don Gregorio Marañón. De sus visitas a las obras de terminación y acondicionamiento, tan gratas y tan instructivas, en las que siempre yo aprendía algo y crecía la amistad, que años después, se consolidó al ser mi mujer sobrina suya y tener una relación familiar ininterrumpida. 
Viejos recuerdos en torno a la construcción...

En el Museo Cajal se pensó, desde el principio, montarlo en un espacio adecuado a los importantes objetos valiosísimos que contenía. Sobre todo, exponer un mueble, en el que se guardaban todas las preparaciones microscópicas de los cortes histológicos del cerebro y los preciosos dibujos hechos por Cajal de las neuronas: verdaderas obras de arte.

De momento y de forma provisional, para evitar pérdidas de mobiliario y material de laboratorio, fotografías, condecoraciones, correspondencia, etc., se instaló, siempre de una forma provisional, pero segura, en el primer tramo del semisótano del edificio, con entrada directa desde la escalera de acceso. 